\title{
STRUCTURE FUNCTIONS IN AN INTERACTING BOSON SYSTEM*
}

\author{
V.K. WONG \\ Department of Physics, University of Michigan, Ann Arbor, Michigan 48104, USA \\ and \\ H. GOULD \\ Department of Physics, Clark University, Worcester, Massachusetts 01610, USA
}

Received 20 March 1972

\begin{abstract}
A microscopic analysis of the dynamic structure factor in a simple interacting Boson system at $T=0$ shows that the coherent two-phonon backflow leads to the breakdown of the one-phonon Feynman relation for the statis structure factor at order $k^{3}$.
\end{abstract}

We report the results of a microscopic analysis of the dynamic structure factor [1] $S(k, \omega)$ and its moments $S_{m}(k)=\int_{0}^{\infty} \mathrm{d} \omega \omega^{m} S(k, \omega)$ for a system of Bosons of mass $m$ at zero temperature and density $n$ to the first approximation beyond the Bogoliubov (zeroth) approximation. The two-body interaction $v$ is characterized by the s-wave scattering length and is taken to be a constant in $k$-space. The small dimensionless parameter $g=4 \pi a m s_{0}$, where $s_{\mathrm{o}}=(4 \pi a n)^{1 / 2} / m$ is the phonon speed in the zeroth approximation. We choose units such that $\hbar=m=s_{\mathrm{o}}=1$.

We write $S(k, \omega)$ as a coherent one-phonon part, $g S_{I}(k, \omega)=Z(k) \delta\left(\omega-\omega_{k}\right)$, and a multi-phonon part $[2,3]$. In order to isolate the effects of backflow, $S_{\mathrm{I}}$ is constructed to exhaust the $f$-sum rule, $g S_{\mathrm{I}}(k)=\frac{1}{2} k^{2}$ thus, $Z(k)=$ $k^{2} / 2 \omega_{k}$ and $\omega_{k}$ is the energy of the one-phonon excitations. The form of the multiphonon term and the $k$-dependence of $S_{m}(k)$ is of interest.

The method of calculation of $S(k, \omega)$ is based on the generalized dielectric formulation [4] of Bose systems and can be outlined as follows. We relate $S(k, \omega)$ to the density-density response function [4] $F(k, \omega) ; S(k, \omega)=$ $-\operatorname{Im} F(k, \omega) / \pi$. The effects of backflow are conveniently taken into account by the identity [4] $g F(k, \omega)=$ $\left(k^{2} / \omega^{2}\right)\left[1+g F^{33}(k, \omega)\right]$ based on the continuity equation, where $F^{33}$ is the longitudinal current response function [4]. A perturbation expansion for $S(k, \omega)$ is developed by expanding $F^{33}, F, S, \omega_{k}^{2}$ and $v$ in powers of $g$, e.g., $g S(k, \omega)=S^{(0)}(k, \omega)+g S^{(1)}(k, \omega)+\ldots ; \omega_{k}^{2}=\omega_{k}^{2}(0)+g \omega_{k}^{2(1)}+\ldots ; v / g=1+g v^{(1)}+\ldots$. We find that to $O\left(g^{\circ}\right)$ $S(k, \omega)$ has only a coherent one-phonon part $S_{I}^{(0)}(k, \omega)=Z^{(0)}(k) \delta\left(\omega-\omega_{k}^{(0)}\right)$, where $Z^{(0)}(k)=k^{2} / 2 \omega_{k}^{(0)}$ and $\omega_{k}^{(0)}=$ $k\left(1+k^{2} / 4\right)^{1 / 2}$. To $O(g)$ we obtain

$$
\begin{aligned}
& F^{(1)}(k, \omega)=k^{2} \omega^{-2}\left(\omega^{2}-\omega_{k}^{2(0)}\right)^{-2} N^{33(1)}(k, \omega) \\
& N^{33(1)}(k, \omega)=\omega^{2} k^{2} v+\frac{1}{2} k^{6}(S-\mu)+\frac{1}{2} \omega k^{4} A+\left(\omega^{2}-k^{4} / 4\right)\left[\frac{1}{2} k^{2}\left(S+M_{2}-\mu\right)-\frac{1}{4} k^{4} n^{\prime}+k \omega\left(\Lambda_{+}^{3}+\Lambda_{-}^{3}\right)+\frac{1}{2} k^{3}\left(\Lambda_{+}^{3}-\Lambda_{-}^{3}\right)\right]+ \\
& +\left(\omega^{2}-k^{4} / 4\right)^{2} F^{33 \mathrm{r}}
\end{aligned}
$$

where the integrals on the right hand side of eq. (2) are given explicitly in ref. [5] with $Q^{ \pm}=\left(\omega-\omega_{p+k}^{(0)}-\omega_{p}^{(0)}\right)^{-1}+$ $\mp\left(\omega+\omega_{p+k}^{(0)}+\omega_{p}^{(0)}\right)^{-1}$. To $O(g)$ the dynamic structure factor is given by $S^{(1)}(k, \omega)=-\operatorname{Im} F^{(1)}(k, \omega) / \pi$. The onephonon contribution $S_{I}^{(1)}$ is simply the expansion ot $O(g)$ of $g S_{1}(k, \omega)$; we find $Z^{(1)}(k)=-k^{2} \omega_{k}^{2(1)} / 4 \omega_{k}^{3(o)}$, where $\omega_{k}^{2(1)}[5]$ and hence $Z(k)$ are not analytic functions of $k$. The multiphonon contribution to $S^{(1)}(k, \omega)$ can be written as a sum of two terms. The coherent two-phonon part $S_{\text {II }}$ is defined as the remaining coherent part

* Supported in part by the Michigan-Memorial Phoenix Project. 
$S_{I I}^{(1)}(k, \omega)=Y^{(1)}(k) \delta\left(\omega-\omega_{k}^{(0)}\right)$

where

$Y^{(1)}(k)=\frac{1}{4}\left(k^{2} / \omega_{k}^{2(o)}\right) \frac{\mathrm{d}}{\mathrm{d} \omega}\left(\omega^{-2} N^{33(1)}\right) \omega_{k}^{(\mathrm{o})}$

and $Y^{(1)}(k)=y_{3} k^{3}+y_{\mathrm{L} 5} k^{5} \ln 1 / k+\ldots$ with $y_{3}=-(7 / 1440) \pi^{-2}$ and $y_{\mathrm{L} 5}=-(3 / 640) \pi^{-2}$. The incoherent two-phonon part $X(k, \omega)$ is found to be

$X^{(1)}(k, \omega)=-\left(k^{2} / \pi \omega^{2}\right)\left(\omega^{2}-\omega_{k}^{2(o)}\right)^{-2} \operatorname{Im} N^{33(1)}(k, \omega)$.

For large $\omega$ and $k \rightarrow 0, X^{(1)}(k, \omega)$ scales as $\left(7 / 120 \pi^{2}\right) \omega^{-3 / 2}\left(k^{2} / \omega\right)^{2}\left[1+O\left(k^{2} / \omega\right)\right]$.

The first-order processes corresponding to $S_{I}^{(1)}, S_{I I}^{(1)}$, and $X^{(1)}$ can be given a simple physical interpretation. $S_{1}^{(1)}$ represents the production of a real phonon to $O(g)$ that exhaust the $f$-sum rule. $S_{(1)}^{(1)}$ corresponds to the production of a real phonon in $O\left(g^{0}\right)$ that is surrounded in $O(g)$ by two virtual phonons, i.e., backflow. $X^{(1)}$ can be interpreted as the production of a virtual phonon that subsequently decays into two real phonons, giving rise to a background.

It can be seen from the above that

$S_{m}^{(1)}(k)=\left(\omega_{k}^{(0)}\right)^{m}(1-m) Z^{(1)}(k)+\left(\omega_{k}^{(0)}\right)^{m} Y^{(1)}(k)+X_{m}^{(1)}(k)$

where $X_{m}^{(1)}(k)=\int_{0}^{\infty} \mathrm{d} \omega \omega^{m} X^{(1)}(k, \omega)=x_{m} k^{4}+\ldots$

represents the contribution from incoherent two-phonon background. From the large $\omega$ behavior of $X^{(1)}(k, \omega)$ we see that $X_{m}^{(1)}(k)$ and hence $S_{m}^{(1)}(k)$ is divergent for $m \geqslant 3$. Thus our perturbation expansion for the moments $S_{m}(k)$ breaks down for $m \geqslant 3$ and our form for $S^{(1)}(k, \omega)$ holds only for $\omega<1 / g$. For $-1 \leqslant m \leqslant 2$ the perturbation expansion of $S_{m}(k)$ is well-defined through $O(g)$, and $x_{-1}=(7 / 7680) \pi^{-2} \ln 1 / k, x_{o}=(7 / 10240) \pi^{-1}, x_{1}=$ $(7 / 1440) \pi^{-2}, x_{2}=(7 / 480) \pi^{-1}$. It is easy to check that $S_{-1}(k)$ and $S_{1}(k)$ are consistent with the compressibility and $f$-sum rules respectively. Since the leading contribution of the coherent two-phonon backflow to $S_{m}^{(1)}(k) \propto$ $k^{3+m}$, the one-phonon Feynman relation for the static structure factor $S_{0}(k), g S_{0}(k)=k^{2} / 2 \omega_{k}$, breaks down at $\mathrm{O}\left(k^{3}\right)$. Note that even with our simple form for $v, S_{\mathrm{o}}(k)$ includes both even and odd terms in $k$ and has an inflection point. We also see that for $m=-1,0,2, S_{m}^{(1)}(k)$ is not an analytic function of $k$, but includes terms $\propto k^{5+m} \ln 1 / k$.

As discussed in ref. [5] the logarithmic term in $S_{m}^{(1)}(k)$ might be expected in superfluid helium. In ref. [2] general arguments for the leading $k^{4}$ dependence of $X_{m}^{(1)}(k)$ were given; we now present similar arguments for the $k^{3+m}$ contribution of the coherent multiphonon backflow which was negelcted in ref. [2]. We write [2] $S_{m}(k)=\sum_{n}\left|\left\langle n\left|\rho_{k}^{+}\right| 0\right\rangle\right|^{2}\left(\omega_{n o}\right)^{m}$. The contribution of the multiphonon backflow can be written

$\sim\left\langle 0\left|\rho_{k}\right| 1\right\rangle\left\langle 1\left|B_{k} \rho_{k}^{+}\right| 0\right\rangle\left(\omega_{10}\right)^{m}$

where $B_{k}$ is the matrix element of the square of the backflow operator $\rho_{k}^{B}=k J_{k}^{B} / \omega$. In the limit $k \rightarrow 0$, the backflow current $J_{k}^{B}$ is finite from hydrodynamical considerations and the excitation energy $\omega$ is finite because of the multiphonons; thus $\rho_{k}^{B} \sim k$ and $B_{k} \sim k^{2}$. It is easy to show [2] that $\left\langle 1\left|\rho_{k}^{+}\right| 0\right\rangle \sim k^{1 / 2}$, and since $\omega_{10} \sim k$ the multiphonon backflow contribution to $S_{m}(k) \propto k^{3+m}$.

\section{References}

[1] R.A. Cowley and A.D.B. Woods, Can. J. Phys. 49 (1971) 177.

[2] A. Miller, D. Pines and P. Nozieres, Phys. Rev. 127 (1962) 1452.

[3] D. Pines and C.-W. Woo, Phys. Rev. Lett. 24 (1970) 1044.

[4] S.-K. Ma, H. Gould and V.K. Wong, Phys. Rev. A3 (1971) 1453.

[5] H. Gould and V.K. Wong, Phys. Rev. Lett. 27 (1971) 301. 